\title{
A logical method for the synthesis of periodic trajectory in a binary dynamical system
}

\author{
G A Oparin, V G Bogdanova and A A Pashinin
}

Matrosov Institute for System Dynamics and Control Theory SB RAS, Lermontov St. 134, Irkutsk, Russia, 664033

bvg@icc.ru

\begin{abstract}
A logic method for structural-parametric synthesis of a binary dynamical system with a given periodic trajectory is proposed. This method provides a constructive solution for the considered problem. The attraction region of such a trajectory must coincide with a given subset of the state space. An additional constraint sets the acceptable time for reaching this trajectory from its attraction region. As admissible structures for dynamical models of the synthesis, we consider the following systems: linear systems, systems with the disjunctive and conjunctive right sides. All conditions of the problem are written in the form of a quantified Boolean formula with subsequent verification of its truth using a specialized solver, which gives values of the required parameters of the dynamical model. The software implementation of the proposed method in the form of a composite service is presented. All stages of the parametric synthesis of a Boolean network based on the proposed method are demonstrated in the example of a one-step linear system.
\end{abstract}

\section{Introduction}

Our research is focused on solving the problem of synthesizing a Boolean network with a given periodic trajectory and a constraint on the time it takes to reach this trajectory.

We use a logic method (the Boolean constraints method [1]) for synthesizing a dynamical model with the required property of cyclicity of a given trajectory. A dynamical property is written as a formula in the applied predicate logic language with bounded quantifiers of existence and universality. The parameters of the Boolean network are selected based on this formal requirement by solving the problem of verifying the truth of a quantified Boolean formula with the simultaneous construction of a constructive solution to the parametric synthesis problem.

The proposed method for the parametric synthesis of a binary dynamic system (BDS) is implemented as a composite service [2] based on our microservice-oriented framework [3]. The microservice-oriented implementation makes it easy to reuse, update, and replace software components and also provides a lightweight messaging protocol for their interaction [4].

The paper is structured as follows. In Section 2, works related to the research topic are considered. Classes of dynamic models and the problem statement of structural-parametric synthesis of BDS with a given periodic trajectory are discussed in Section 3. In Section 4, a method for solving the structuralparametric synthesis problem for a linear BDS is proposed. The method implementation is given in Section 5. In Section 6, the synthesis of the Boolean network is considered in the example of a onestep linear system using the proposed method. The final section 7 summarizes the research results and lists the advantages of the proposed method.

Copyright $(C 2021$ for this paper by its authors. Use permitted under Creative Commons License Attribution 4.0 International (CC BY 4.0). 


\section{Related work}

Periodic trajectories (cycles) are a significant component of the phase portrait of any BDS due they determine the long-term behavior of the system after the completion of transient processes. A large number of studies (for example, [1,5-12]) are devoted to the search for cycles of unit length (equilibrium states, fixed points). From our point of view, this problem has been fully solved. Methods for searching for the cycles of a fixed but greater than one length are considered in [1, 13-15]. Computationally, this problem is more complicated than finding fixed points. Even more great difficulties arise during constructing the entire set of cycles [16-18], especially for systems with a high dimension of the state vector. The problems listed above are related to BDS analysis problems.

The inverse problem is equally important. This problem is the structural-parametric synthesis of a dynamic model of a Boolean network with a given behavior in a steady-state and additional constraints on the parameters of the transient process. The most active research in this direction is observed when the required set of one-point attractors is given. In particular, two algorithms for the synthesis of a Boolean network are proposed in [19]. These algorithms are based on a random search procedure. In the first algorithm, a predictor set is randomly generated for each node. This set satisfies the specified constraints on the number of elements in the predictors. In the second algorithm, a transition diagram is randomly assigned with a given set of attractors. Next, the constraints are checked using the obtained set of predictors. In [20], the problem of constructing large-scale networks with given attractors and their maximum domains of attraction is considered. In [21], a particular case of the synthesis of a Boolean network for a given set of attractors is considered when each Boolean function represents the conjunction or disjunction of literals. All the above methods use, to some extent, a Boolean network graph.

In [22], to solve the synthesis problem, a method of reduction (simplification) of a Boolean network graph was proposed. Additionally, this method was demonstrated on a biological example of an apoptosis gene regulatory network. The problem of structural-parametric synthesis of BDS with a given periodic trajectory of length greater than one and a constraint on the nature of convergence to this trajectory is not considered in this article. However, the importance of such a problem is noted. The author considers its solution as one of the directions for further research. Issues of synthesis of cyclic finite automata representing a specific class of technologic machines of discrete action (hydraulic and pneumatic drives) are considered in [23]. A method is proposed for describing the operation of cyclic automata using cyclograms with their further transformation to an accepted automaton description in the form of transition tables. It should be noted that cyclicity is the main mode of functioning of a large number of objects, for example, control systems for industrial robots [24], systems for correcting human gait [25], etc. In this regard, it becomes necessary to synthesize discrete control devices (so-called signal distributors) that provide the specified cyclicity parameters.

\section{Mathematical model and problem statement}

The solution to the problem of synthesis of a BDS, the phase portrait of which contains only one given periodic trajectory possessing the property of attraction [1], is considered within the framework of three classes of dynamic models of the following form:

$$
X(t+1)=A \otimes X(t) \circ U, X(0)=x^{0},
$$

where $t=0,1,2, \ldots$ is the discrete time, $X(t)=\operatorname{col}\left(x_{1}(t), x_{2}(t), \ldots x_{n}(t)\right)$ is the state vector, $X(t) \in B^{n}, B=\{0,1\}, B^{n}$ is the state space of the system (1), $U=\operatorname{col}\left(u_{1}, u_{2}, \ldots, u_{n}\right)$ is the constant binary control vector, $A$ is a binary matrix of the dimension $n \times n, \otimes$ is the symbol of the operation of multiplying a matrix by a vector, which can take three values, namely $*, \nabla$, and $\Delta$, which are symbols of Boolean, conjunctive and disjunctive multiplication, respectively; the symbol $\circ$ means the operation of componentwise addition $\bmod 2$, disjunction or conjunction of two Boolean vectors, depending on the value of the operation $\otimes$. 
Let us define each of the listed operations as follows:

$$
\begin{aligned}
& (A * X)_{i} \circ U_{i}=\sum_{j=1}^{n}\left(a_{i j} \wedge x_{j}\right) \oplus u_{i} \\
& (A \Delta X)_{i} \circ U_{i}=\bigvee_{j=1}^{n}\left(a_{i j} \wedge x_{j}\right) \vee u_{i} \\
& (A \nabla X)_{i} \circ U_{i}=\wedge_{j=1}^{n}\left(a_{i j} \vee x_{j}\right) \wedge u_{i}
\end{aligned}
$$

Here symbols $\Sigma, \vee, \wedge$ mean the Boolean operation of addition mod2, disjunction and conjunction. The operation $*$ defines a linear BDS. The operation $\nabla$ defines BDS with the disjunctive right side and the operation $\Delta$ - BDS with the conjunctive right side. For the sake of brevity, the state vector $X(t)$ will be written as $x^{t}, t=0,1,2, \ldots$

We define a cyclic trajectory of length $m$ as a sequence of states $C=\left(c^{1}, \ldots, c^{m}\right)$ so that system (1) passes from the state $c^{i}$ to the state $c^{i+1}(i=\overline{1, m-1})$, and from the state $c^{m}$ a transition to the state $c^{1}$ takes place.

It is required for system (1) to find a matrix $A^{*}$ and a control vector $U^{*}$ such that the system

$$
X(t+1)=A^{*} \otimes X(t) \circ U^{*}
$$

had a single cyclic trajectory $C$ and for any state $x^{0} \in B^{n}$ system (2) would go to one of the states of the set $C$ in one step, that is, the following condition would be satisfied:

$$
x^{1}=A^{*} \otimes x^{0} \circ U^{*} \in C .
$$

In this case, $x^{0}$ plays the role of a control parameter that ensures the start of a cyclic trajectory from a given state.

Another formulation of the problem is that the set of possible initial states is bounded by the condition $x^{0} \in B^{n} \backslash\{0\}$, where $\{0\}$ denotes the zero state. This limitation makes it possible to solve the posed problem with admissible constant control $U=0$.

\section{Solution method}

Without loss of generality, consider the problem of synthesizing the characteristic matrix $A$ and the control vector $U$ for a linear BDS of the form (1).

It is known that the linear system of equations (1) with an initial state $x^{0} \in B^{n}$ in a one-step transition is equivalent to one Boolean equation

$$
L\left(x^{0}, x^{1}, A, U\right)=\vee_{i=1}^{n}\left(x_{i}^{1} \oplus \sum_{j=1}^{n} a_{i j} \wedge x_{j}^{0} \oplus u_{i}\right)=0 .
$$

Each pair of neighboring states from the set $C$, according to the definition of a cyclic trajectory, must satisfy the following system of Boolean equations:

$$
\begin{gathered}
c^{i+1}=A^{*} c^{i} \oplus U, i=\overline{1, m-1} \\
c^{1}=A^{*} c^{m} \oplus U
\end{gathered} .
$$

According to (3), the system of equations (4) is equivalent to one Boolean equation 


$$
\Phi_{1}(A, U)=\bigvee_{i=1}^{m-1} L\left(x^{0}=c^{i}, x^{1}=c^{i+1}, A, U\right) \vee L\left(x^{0}=c^{m}, x^{1}=c^{1}, A, U\right)=0 .
$$

It is assumed that the set of states $C$ of a cyclic trajectory is determined by solutions of the Boolean equation

$$
\Phi_{2}\left(x^{1}\right)=0 .
$$

The condition $x^{0} \in B^{n} \backslash\{0\}$ is equivalent to the following Boolean equation:

$$
\Phi_{3}\left(x^{0}\right)=\bar{x}_{1}^{0} \wedge \bar{x}_{2}^{0} \wedge \ldots \wedge \bar{x}_{n}^{0}=0
$$

Following the Boolean constraints method [1] and the definitions introduced above, the conditions of the posed problem are written in the form of the following formulas in the language of predicate logic with bounded quantifiers of existence and universality:

$$
\begin{gathered}
\left(\exists A, U: \bar{\Phi}_{1}(A, U)\right)\left(\forall x^{0}\right)\left(\exists x^{1}: \bar{L}\left(x^{0}, x^{1}, A, U\right)\right)\left(\bar{\Phi}_{2}\left(x^{1}\right)\right) \\
\left(\exists A, U: \bar{\Phi}_{1}(A, U)\right)\left(\forall x^{0}: \bar{\Phi}_{3}\left(x^{0}\right)\right)\left(\exists x^{1}: \bar{L}\left(x^{0}, x^{1}, A, U\right)\right)\left(\bar{\Phi}_{2}\left(x^{1}\right)\right)
\end{gathered}
$$

where $\bar{L}\left(x^{0}, x^{1}, A, U\right), \bar{\Phi}_{1}(A, U), \bar{\Phi}_{2}\left(x^{1}\right), \bar{\Phi}_{3}\left(x^{0}\right)$ are characteristic functions of Boolean constraints $(3,5-7)$, respectively. The corresponding to (8) quantified Boolean formulas have the following form:

$$
\begin{gathered}
(\exists A, U)\left(\forall x^{0}\right)\left(\exists x^{1}\right)\left(\bar{\Phi}_{1}(A, U) \wedge\left(\bar{L}\left(x^{0}, x^{1}, A, U\right) \wedge \bar{\Phi}_{2}\left(x^{1}\right)\right)\right) \\
(\exists A, U)\left(\forall x^{0}\right)\left(\exists x^{1}\right)\left(\bar{\Phi}_{1}(A, U) \wedge\left(\Phi_{3}\left(x^{0}\right) \vee \bar{L}\left(x^{0}, x^{1}, A, U\right) \wedge \bar{\Phi}_{2}\left(x^{1}\right)\right)\right)
\end{gathered}
$$

where $A, U, x^{0}, x^{1}$ are sets of the following subject variables:

$$
\begin{gathered}
A=\left(a_{11}, a_{12}, \ldots, a_{1 n}, \ldots, a_{n 1}, a_{n 2}, \ldots, a_{n n}\right), \\
U=\left(u_{1}, u_{2}, \ldots, u_{n}\right), \\
x^{0}=\left(x_{1}^{0}, x_{2}^{0}, \ldots, x_{n}^{0}\right), x^{1}=\left(x_{1}^{1}, x_{2}^{1}, \ldots, x_{n}^{1}\right) .
\end{gathered}
$$

The total number of subject variables is $n^{2}+3 n$.

If the quantified Boolean formulas (9) corresponding to the problem statements are TRUE, then a solution (at least one matrix $A^{*}$ and vector $U^{*}$ ) exists. Otherwise, the problem has no solution.

\section{Method implementation}

The proposed method for the parametric synthesis of BDS is implemented as a composite service [26] based on the multiagent platform HPCSOMAS-MSC [3]. This approach makes it possible to develop a distributed scalable system whose components are microservices, which may be implemented using 
heterogeneous technologies and paradigms [2]. The microservice architecture supports modularity, encapsulation, partial deployment, and decentralized control, which improves the fault tolerance in a distributed multiagent system [27]. The distribution of modules on different server nodes and an independent language of interaction allows the using various programming languages and the developing modules for multiple operating systems. This approach is convenient for solving the considered problem of parametric synthesis since it provides the possibility of using previously developed by authors or free software, implemented as microservices, to create a Boolean model, check its truth, and post-process the results. The scheme of composite service for the parametric synthesis of BDS is shown in Figure 1.

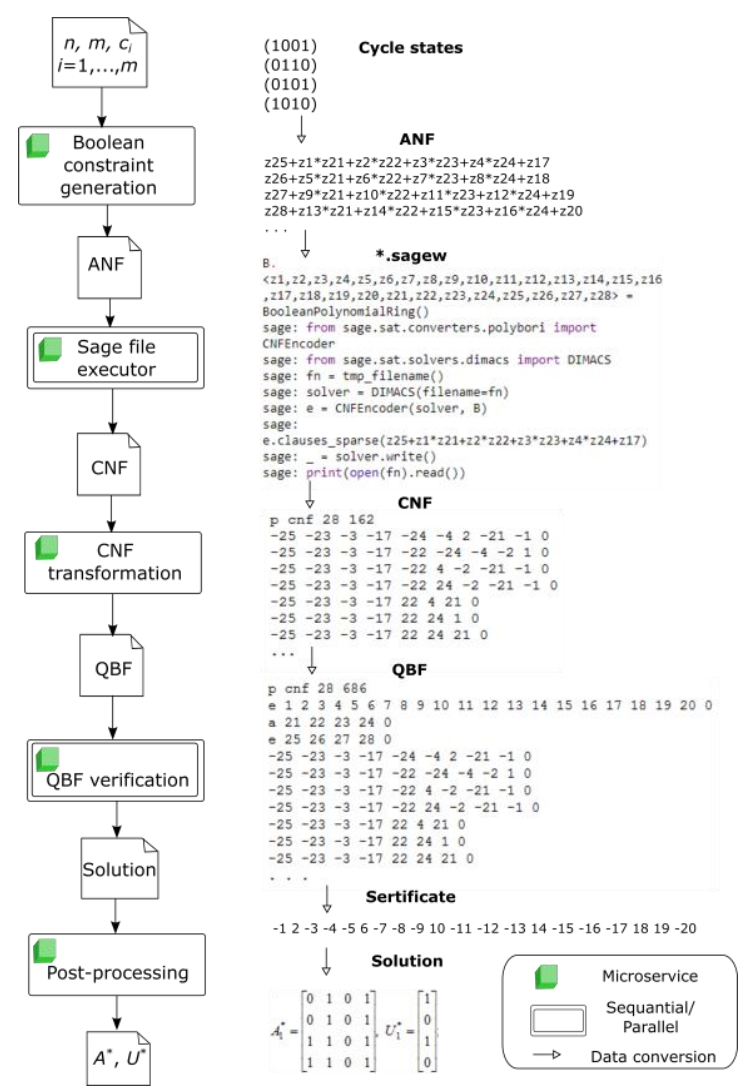

Figure 1. Composite service scheme.

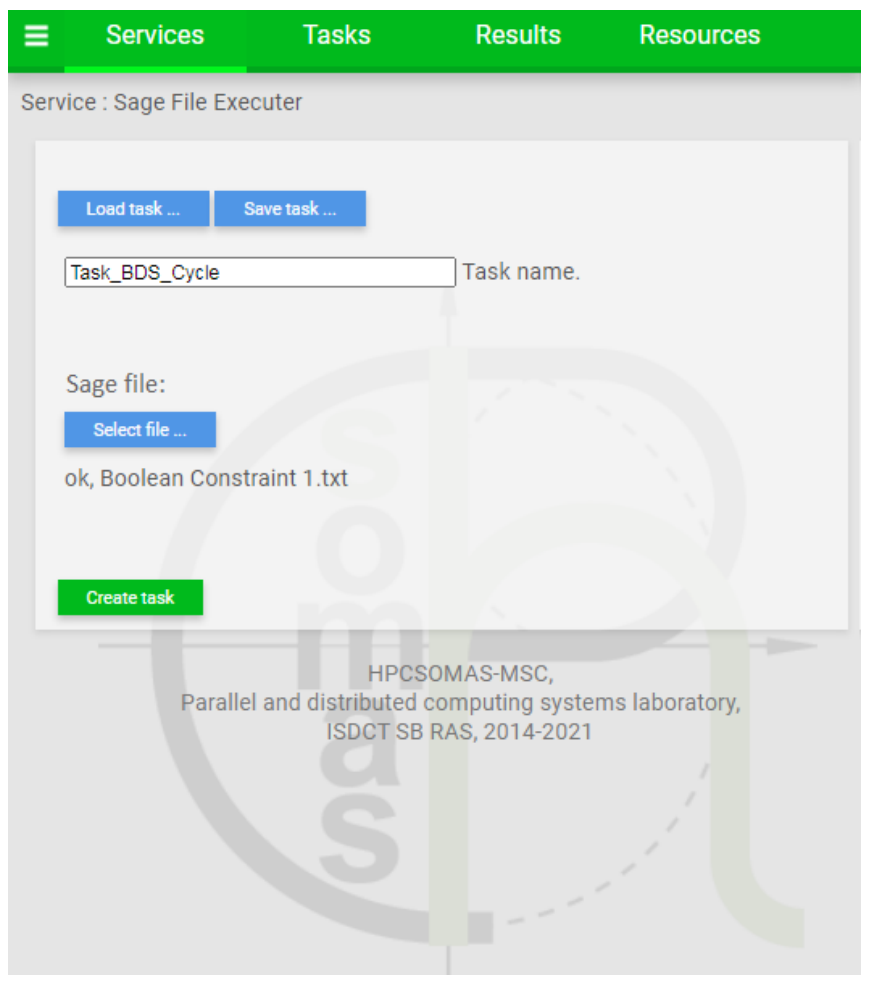

Figure 2. The web interface of the SFE service.

The atomic microservices included in this service are launched when the data is ready by the HPCSOMAS agent [28] installed on the corresponding server node. These microservices have program and web interfaces and can work autonomously. The composite service automates the following stages of solving the problem of parametric synthesis of BDS:

- Generating Boolean constraints $(3,4,6)$ based on user-specified states of a periodic trajectory. These constraints are generated in an algebraic normal form (ANF).

- Generating the final formula (4) corresponding to the problem statement. With the help of the microservice Sage File Executor (SFE), files of the ".sagews" type are first generated for constraints $\bar{L}\left(x^{0}, x^{1}, A, U\right), \bar{\Phi}_{1}(A, U)$, and $\bar{\Phi}_{2}\left(x^{1}\right)(3,4,6)$, and then the converter ANF $=0$ $\rightarrow \mathrm{CNF}=1$ [29] is launched to obtain CNF in DIMACS format [30]. The received CNFs are combined. The web interface of the SFE microservice is shown in Figure 2. 
- Converting CNF. Constraints $\Phi_{3}\left(x^{1}\right)$ are included in CNF, and the Plaistid-Greenbaum transformation [31] is used if $x^{0} \in B^{n} \backslash\{0\}$ (the user can specify the exception of the zero state $\{0\}$ when setting the problem). If necessary, the Zeitin transformation is applied [32]. For the final formation of QBF in the QDIMACS format [33], existential and universality quantifiers are added following formulas (9).

- Verifying the truth of the QBF. For small-scale problems, the truthiness of formulas (9) is performed using the QSAT solver DepQBF [34] with the "issue a certificate" option set [35]. In our case, the certificate is the matrix $A$ and the vector $U$. For calculating one more solution (if it exists), a clause $\bigvee_{i=1}^{n} \bigvee_{j=1}^{n} \bar{a}_{i j}^{*} \vee \bigvee_{i=1}^{n} \bar{u}_{i}^{*}$ is added to the final formula. This clause corresponds to the constraint that excludes the repeated finding of the obtained solution $A^{*}, U^{*}$. After updating this formula, the DepQBF solver is run again. For problems of large dimensions, the previously developed parallel solver Hpcqall [36] is used.

- Post-processing of the obtained results. If a solution to the problem is found, the matrix $A^{*}$ and vector $U^{*}$ are formed using the certificate issued by DepQBF.

\section{Illustrative examples}

Let us consider in detail all the stages of the parametric synthesis of a Boolean network using two simple examples of a one-step linear system of dimension $n=4$ with cyclic trajectories including two and four states. The property of attraction to a cycle in one step is a strong requirement; however, for both examples, it was possible to obtain a constructive solution to the problem of synthesizing a given periodic trajectory.

\subsection{Example 1}

Let $n=4, x^{0} \in B^{n}$, and the set $C$ includes two states $(m=2): c^{1}=\operatorname{col}(1001), c^{2}=\operatorname{col}(0110)$.

Let $Z=\left(z_{1}, z_{2}, \ldots, z_{28}\right)$ be an ordered set of Boolean variables, $z_{i} \in B^{2}$ for all $i=\overline{1,28}$. We use the following encoding of subject variables, namely elements of the matrix $A$ and vectors $U, x^{0}$ and $x^{1}$ :

$$
A=\left[\begin{array}{cccc}
z_{1} & z_{2} & z_{3} & z_{4} \\
z_{5} & z_{6} & z_{7} & z_{8} \\
z_{9} & z_{10} & z_{11} & z_{12} \\
z_{13} & z_{14} & z_{15} & z_{16}
\end{array}\right], U=\left[\begin{array}{c}
z_{17} \\
z_{18} \\
z_{19} \\
z_{20}
\end{array}\right], \begin{aligned}
& x^{0}=\left(z_{21}, z_{22}, z_{23}, z_{24}\right), \\
& x^{1}=\left(z_{25}, z_{26}, z_{27}, z_{28}\right)
\end{aligned} .
$$

Boolean constraints $(3,4,6)$ are conveniently represented in the ANF:

1) $x^{1}=A^{*} x^{0} \oplus U:$

$$
\begin{aligned}
& z_{25}=z_{1} \cdot z_{21} \oplus z_{2} \cdot z_{22} \oplus z_{3} \cdot z_{23} \oplus z_{4} \cdot z_{24} \oplus z_{17} \\
& z_{26}=z_{5} \cdot z_{21} \oplus z_{6} \cdot z_{22} \oplus z_{7} \cdot z_{23} \oplus z_{8} \cdot z_{24} \oplus z_{18} \\
& z_{27}=z_{9} \cdot z_{21} \oplus z_{10} \cdot z_{22} \oplus z_{11} \cdot z_{23} \oplus z_{12} \cdot z_{24} \oplus z_{19} \\
& z_{28}=z_{13} \cdot z_{21} \oplus z_{14} \cdot z_{22} \oplus z_{15} \cdot z_{23} \oplus z_{16} \cdot z_{24} \oplus z_{20}
\end{aligned}
$$

2) $c^{2}=A * c^{1} \oplus U, c^{1}=A * c^{2} \oplus U:$

$$
\begin{array}{rlll}
z_{1} \oplus z_{4} \oplus z_{17}=0 & z_{2} \oplus z_{3} \oplus z_{17}=1 & z_{9} \oplus z_{12} \oplus z_{19}=1 & z_{10} \oplus z_{11} \oplus z_{19}=0 \\
z_{5} \oplus z_{8} \oplus z_{18}=1 & z_{6} \oplus z_{7} \oplus z_{18}=0 & z_{13} \oplus z_{16} \oplus z_{20}=0 & z_{14} \oplus z_{15} \oplus z_{20}=1
\end{array} ;
$$


3) $x^{1} \in C:\left(z_{25} \oplus z_{28}\right)=0,\left(z_{26} \oplus z_{27}\right)=0,\left(z_{25} \oplus \bar{z}_{26}\right)=0,\left(z_{27} \oplus \bar{z}_{28}\right)=0$.

Using the composite HPCSOMAS service, we obtain QBF (9) and verify its truth. The problem has eight solutions (Figure 3).

$$
\begin{aligned}
& A_{1}^{*}=\left[\begin{array}{llll}
0 & 1 & 0 & 0 \\
0 & 1 & 0 & 0 \\
0 & 1 & 0 & 0 \\
0 & 1 & 0 & 0
\end{array}\right], U_{1}^{*}=\left[\begin{array}{l}
0 \\
1 \\
1 \\
0
\end{array}\right] ; A_{2}^{*}=\left[\begin{array}{llll}
1 & 0 & 0 & 0 \\
1 & 0 & 0 & 0 \\
1 & 0 & 0 & 0 \\
1 & 0 & 0 & 0
\end{array}\right], U_{2}^{*}=\left[\begin{array}{l}
1 \\
0 \\
0 \\
1
\end{array}\right] ; A_{3}^{*}=\left[\begin{array}{llll}
0 & 0 & 0 & 1 \\
0 & 0 & 0 & 1 \\
0 & 0 & 0 & 1 \\
0 & 0 & 0 & 1
\end{array}\right], U_{3}^{*}=\left[\begin{array}{l}
1 \\
0 \\
0 \\
1
\end{array}\right] \\
& A_{4}^{*}=\left[\begin{array}{llll}
0 & 0 & 1 & 0 \\
0 & 0 & 1 & 0 \\
0 & 0 & 1 & 0 \\
0 & 0 & 1 & 0
\end{array}\right], U_{4}^{*}=\left[\begin{array}{l}
0 \\
1 \\
1 \\
0
\end{array}\right], A_{5}^{*}=\left[\begin{array}{llll}
0 & 1 & 1 & 1 \\
0 & 1 & 1 & 1 \\
0 & 1 & 1 & 1 \\
0 & 1 & 1 & 1
\end{array}\right], U_{5}^{*}=\left[\begin{array}{l}
1 \\
0 \\
0 \\
1
\end{array}\right], A_{6}^{*}=\left[\begin{array}{llll}
1 & 1 & 0 & 1 \\
1 & 1 & 0 & 1 \\
1 & 1 & 0 & 1 \\
1 & 1 & 0 & 1
\end{array}\right], U_{6}^{*}=\left[\begin{array}{l}
0 \\
1 \\
1 \\
0
\end{array}\right] ; \\
& A_{7}^{*}=\left[\begin{array}{llll}
1 & 1 & 1 & 0 \\
1 & 1 & 1 & 0 \\
1 & 1 & 1 & 0 \\
1 & 1 & 1 & 0
\end{array}\right], U_{7}^{*}=\left[\begin{array}{l}
1 \\
0 \\
0 \\
1
\end{array}\right] A_{8}^{*}=\left[\begin{array}{llll}
1 & 0 & 1 & 1 \\
1 & 0 & 1 & 1 \\
1 & 0 & 1 & 1 \\
1 & 0 & 1 & 1
\end{array}\right], U_{8}^{*}=\left[\begin{array}{l}
0 \\
1 \\
1 \\
0
\end{array}\right] .
\end{aligned}
$$

Figure 3. Solutions for example 1.

For the first of the obtained solutions, the scalar representation of the BDS (2) has the following form:

$$
x_{1}^{t+1}=x_{2}^{t}, x_{2}^{t+1}=x_{2}^{t} \oplus 1, \quad x_{3}^{t+1}=x_{2}^{t} \oplus 1, \quad x_{4}^{t+1}=x_{2}^{t} .
$$

The transition diagram of system (2) is shown in Figure 4.

\subsection{Example 2}

Let us perform a parametric synthesis of a Boolean network using a one-step linear system of dimension $n=4$ for the case when $x^{0} \in B^{n}$ and the set $C$ includes four states $(m=4)$ :

$$
c^{1}=\operatorname{col}(1001), c^{2}=\operatorname{col}(0110), c^{3}=\operatorname{col}(0101), c^{4}=\operatorname{col}(1010) .
$$

In this case, the Boolean constraint (3) remains the same and has the form (10). For $m=4$, the system (4) is written as follows:

$$
c^{2}=A * c^{1} \oplus U, \quad c^{3}=A * c^{2} \oplus U, \quad c^{4}=A * c^{3} \oplus U, \quad c^{1}=A * c^{4} \oplus U .
$$

For the given states of the set $C$, constraint (11) has the following form:

$$
\begin{array}{cccc}
z_{1} \oplus z_{4} \oplus z_{17}=0 & z_{2} \oplus z_{3} \oplus z_{17}=0 & z_{2} \oplus z_{4} \oplus z_{17}=1 & z_{1} \oplus z_{3} \oplus z_{17}=1 \\
z_{5} \oplus z_{8} \oplus z_{18}=1 & z_{6} \oplus z_{7} \oplus z_{18}=1 & z_{6} \oplus z_{8} \oplus z_{18}=0 & z_{5} \oplus z_{7} \oplus z_{18}=0 \\
z_{9} \oplus z_{12} \oplus z_{19}=1 & z_{10} \oplus z_{11} \oplus z_{19}=0 & z_{10} \oplus z_{12} \oplus z_{19}=1 & z_{9} \oplus z_{11} \oplus z_{19}=0 \\
z_{13} \oplus z_{16} \oplus z_{20}=0 & z_{14} \oplus z_{15} \oplus z_{20}=1 & z_{14} \oplus z_{16} \oplus z_{20}=0 & z_{13} \oplus z_{15} \oplus z_{20}=1
\end{array} .
$$

The Boolean constraint $x^{1} \in C$ is written as follows: $z_{25} \oplus z_{26}=1, \quad z_{27} \oplus z_{28}=1$.

Similar to example 1, we get QBF (9) in QDIMACS format, using the HPCSOMAS service. When checking the truth of this QBF, 16 solutions were obtained. For the first of the obtained solutions 


$$
A_{1}^{*}=\left[\begin{array}{llll}
0 & 1 & 0 & 1 \\
0 & 1 & 0 & 1 \\
1 & 1 & 0 & 1 \\
1 & 1 & 0 & 1
\end{array}\right], U_{1}^{*}=\left[\begin{array}{l}
1 \\
0 \\
1 \\
0
\end{array}\right],
$$

the scalar representation of the BDS (2) has the following form:

$$
x_{1}^{t+1}=x_{2}^{t} \oplus x_{4}^{t} \oplus 1 \quad x_{2}^{t+1}=x_{2}^{t} \oplus x_{4}^{t} \quad x_{3}^{t+1}=x_{1}^{t} \oplus x_{2}^{t} \oplus x_{4}^{t} \oplus 1 \quad x_{4}^{t+1}=x_{1}^{t} \oplus x_{2}^{t} \oplus x_{4}^{t} .
$$

The transition diagram of system (2) is shown in Figure 5.

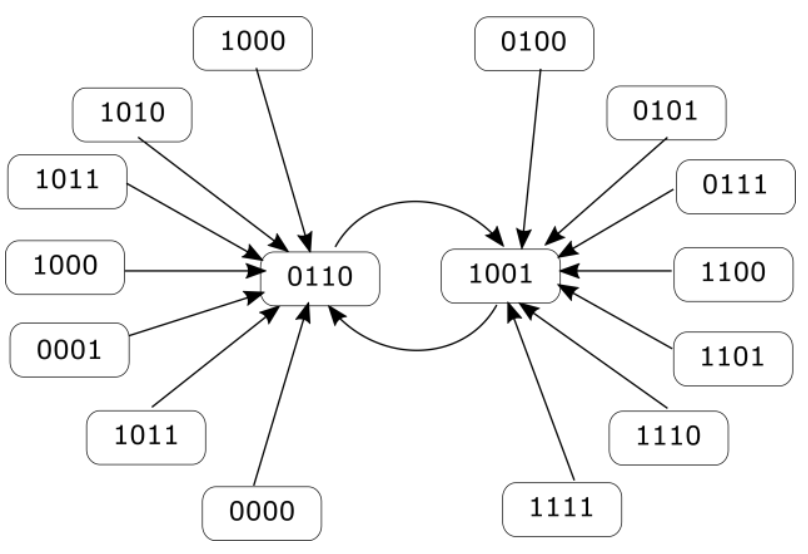

Figure 4. The transition diagram to example 1.

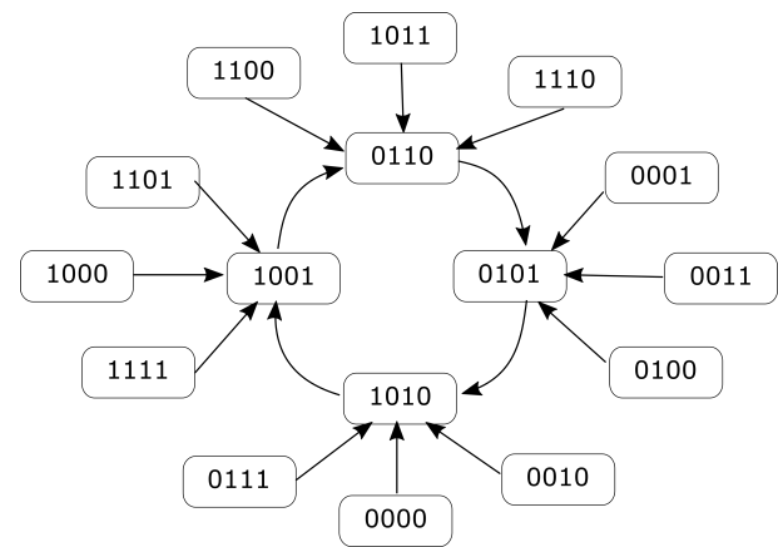

Figure 5. The transition diagram to example 2.

In both examples, we used the condition $x^{0} \in B^{n}$, which means that the zero state $\{0\}$ belongs to the attraction region of the cycle. Therefore, the acceptable values of $U$ are bounded by the states of set $C$. It is evident from the analysis of the solutions in both examples.

Additionally, it should be noted that the transition diagram in each of the above examples is structurally equivalent in the sense that each state of the periodic trajectory has the same number of predecessors that do not belong to the cycle.

\section{Conclusion}

A logic method is proposed for solving the problem of structural-parametric synthesis of BDS with the required periodic trajectory and the time to reach this trajectory from the region of its attraction. Service-oriented automation tools are developed for all stages of solving this problem based on the proposed method.

If necessary, the property formula can accommodate multi-step transitions, restrictions on the trajectory length within the attraction region, and other requirements. The formal specification of a property (in contrast to its verbal description) does not allow the interpretation ambiguity of the meaning of a property. Such specification allows, through a series of sequential formal transformations, to form a model of a dynamic property in the form of a Boolean constraint (a quantified Boolean formula). This model satisfies both the logic specification of the property and the equations of binary system dynamics.

The novelty of obtained results consists in a highly formalized approach to solving problems of qualitative analysis and parametric synthesis of BDS based on the Boolean constraints method and its implementation using microservice technology. The practical application of the developed tools is due to the widespread use of BDS as models of the objects under study in various subject areas. For 
example, the BDS synthesized in this study can be attributed to the so-called signal distributors since the sequence of states (signals) formed at the BDS outputs is applicable in biological applications, in industrial robot control systems, in human gait correction systems, etc.

\section{Acknowledgments}

The study was supported by the Ministry of Science and Higher Education of the Russian Federation, project «Technologies for the development and analysis of subject-oriented intelligent group control systems in non-deterministic distributed environments». The authors would like to thank Irkutsk Supercomputer Center of SB RAS for providing the access to HPC-cluster "Akademik V.M. Matrosov" [37].

\section{References}

[1] Oparin G, Bogdanova V and Pashinin A 2019 Qualitative analysis of autonomous synchronous binary dynamic systems MESA 10(3) 407-419

[2] Huf A and Siqueira F 2019 Composition of heterogeneous web services: A systematic review Journal of Network and Computer Applications 143 89-110

[3] Oparin G A, Bogdanova V G, Pashinin A A and Gorsky S A 2019 Microservice-oriented approach to automation of distributed scientific computations Proceedings of the $42 \mathrm{st}$ International Convention on Information and Communication Technology, Electronics and Microelectronics (MIPRO), Opatija, 2019 pp 253-258

[4] Newman S 2015 Building Microservices (O’Reilly)

[5] Azuma S, Yoshida T and Sugie T 2017 Structural monostability of activation-inhibition Boolean networks IEEE Transactions on control of network systems 4(2) 179-190

[6] Coënt A Le, Friborg L and Soulat R 1016 Compositional analysis of Boolean networks using local fixed-point iterations Reachability Problems. RP 2016. Lecture Notes in Computer Science, vol 9899 ed K Larsen, I Potapov and J Srba (Cham: Springer)

[7] Paulevé L and Richard A 2012 Static analysis of Boolean networks based on interaction graphs: A Survey Electronic Notes in Theoretical Computer Science 284 93-104

[8] Tamura T and Akutsu T 2009 Detecting a singleton attractor in a Boolean network utilizing sat algorithms IECE Transactions on Fundamentals of Electronics, Communications and Computer Sciences E92-A(2) 493-501

[9] Veliz-Cuba A, Aguilar B, Hincelmann F and Laubenbacher R 2014 Steady state analysis of Boolean molecular network models via model reduction and computational algebra $B M C$ Bioinformatics 15(1) article no. 221

[10] Veliz-Cuba A and Laubenbacher R 2012 On the computation of fixed points in Boolean networks J. Appl. Math. Comput. 39 145-153

[11] Aracena J, Richard A and Salinas L 2014 Maximum number of fixed points in AND-OR-NOT networks J. Comput. Syst. Sci. 80 1175-1190

[12] Dubrova E and Teslenko M 2011 A SAT-based algorithm for finding attractors in synchronous Boolean networks in IEEE/ACM Transactions on Computational Biology and Bioinformatics 8(5) 1393-1399

[13] Fornasini E and Valcher M E 2013 On the periodic trajectories of Boolean control networks Automatica 49(5) 1506-1509

[14] Defant C 2017 Binary codes and period-2 orbits of secuential dynamical systems Discrete Math. Theor. Comput. 19(3) DMTCS-10(2017)10

[15] Aledo J A, Martinez S and Valverde J C 2013 Updating method for the computation of orbits in parallel and sequential dynamical systems Int. J. Comput. Math. 90 1796-1808

[16] Aledo J A, Diaz L G, Martinez S and Valverde J C 2018 Maximum number of periodic orbits in parallel dynamical systems Inf. Sci. 468 63-71

[17] Dubrova E and Teslenko M 2016 A SAT-based algorithm for finding short cycles in shift register based stream ciphers in IACR Cryptology ePrint Archive 106 
[18] Bogdanova V G, Gorsky S A and Pashinin A A 2020 HPC-based parallel software for solving applied Boolean satisfiability problems Proceedings of the 43rd International Convention on Information and Communication Technology, Electronics and Microelectronics (MIPRO), IEEE, pp 1231-1236

[19] Pal R, Ivanov I, Datta A, Bittner M L and Dougherty E R 2005 Generating Boolean networks with a prescribed attractor structure Bioinformatics 21(21) 4021-4025

[20] Pan J, Feng Je, Meng M and Zhao J 2018 Design of Large-scale Boolean Networks Based on Prescribed Attractors Int. J. Control Autom. Syst. 16(3) 1120-1128

[21] Jiang H, Tamura T, Ching W-K and Akutsu T 2013 On the complexity of inference and completion of Boolean networks from given singleton attractors IEICE Transactions on Fundamentals of Electronics, Communications and Computer Sciences, E96-A(11) 22652274

[22] Kobayashi K 2019 Design of fixed points in Boolean networks using feedback vertex sets and model reduction Complexity 2019 C(2019)9261793

[23] Yuditskii S A 1969 Utilization of cyclogram language in synthesis of cyclic automata Autom. Remote Control 30(1) 82-92

[24] Crama Y, Kats V, Klundert J and Levner E 2000 Cyclic scheduling in robotic flowshops Annals of Operations Research 96 97-124

[25] Jasni F, Hamzaid NA, Syah NE M, Chung T Y and Osman NA A 2017 Analysis of iInterrelationships among voluntary and prosthetic leg joint parameters using cyclograms Front. Neurosci. 11 FNINS-11(2017)230

[26] Oparin G A, Bogdanova V G and Pashinin A A 2020 Automated tools for the development of microservice compositions for hybrid scientific computations Proceedings of the 2nd International Workshop on Information, Computation, and Control Systems for Distributed Environments, Irkutsk, Russia pp 201-213

[27] Khan Z A, Shahid S, Ahmad H F, Ali A and Suguri H 2005 Decentralized architecture for fault tolerant multi agent system Proceedings Autonomous Decentralized Systems (ISADS 2005), pp 167-174

[28] Pashinin A and Bogdanova V 2020 Application of user dew agent in hybrid-computing environments Proceedings of the 1st International Workshop on Advanced Information and Computation Technologies and Systems (AICTS 2020), Irkutsk, Russia, December 7-11, 2020, pp 135-145

[29] SageMath. [Online]. Available: https://www.sagemath.org/

[30] Satisfiability suggested format. [Online]. Available: http://beyondnp.org/static/media/uploads/docs/satformat.pdf [online, accessed: 01.02.2021].

[31] Plaisted D A and Greenbaum S 1986 A Structure Preserving Clause Form Translation J. Symbolic Computation 2(3) 293-304

[32] Tseitin G S 1983 On the complexity of derivation in propositional calculus Automation of Reasoning. Symbolic Computation (Artificial Intelligence) ed J H Siekmann and G Wrightson (Berlin, Heidelberg: Springer) pp 466-483

[33] QDIMACS. [Online] Available: http://www.qbflib.org/qdimacs.html

[34] Lonsing F and Biere A 2010 DepQBF: A Dependency-Aware QBF Solver Journal of Satisfiability, Boolean Modeling and Computation 9 71-76

[35] DepQBF. [Online]. Available: https://lonsing.github.io/depqbf/ [online, accessed: 01.02.2021]

[36] Bogdanova V G and Gorsky S A 2019 Multiagent technology for parallel implementation of Boolean constraint method for qualitative analysis of binary dynamic systems Proceedings of 42st International Convention on Information and Communication Technology, Electronics and Microelectronics (MIPRO), IEEE, pp 1043-1048

[37] Irkutsk Supercomputer Centre of SB RAS. [Online]. Available: http://hpc.icc.ru 